\title{
A Family of Delayed Rectifier Kv1 cDNAs Showing Cell Type-Specific Expression in the Squid Stellate Ganglion/Giant Fiber Lobe Complex
}

\author{
Joshua J. C. Rosenthal, Taylor I. Liu, and William F. Gilly \\ Hopkins Marine Station, Department of Biological Sciences, Stanford University, Pacific Grove, California 93950
}

Squid giant axons are formed by giant fiber lobe (GFL) neurons of the stellate ganglion (SG). Other large motoneurons in the SG form a parallel system. A small family of cDNAs (SqKv1A-D) encoding Kv1 $\alpha$-subunits was identified in a squid (Loligo opalescens) SG/GFL library. Members have distinct 5' untranslated regions (UTRs) and initial coding regions, but beyond a certain point (nucleotide 34 of SqKv1A) only nine differences exist. 3' UTRs are identical. Predicted $\alpha$-subunits are nearly identical, and only the $\mathrm{N}$ termini differ significantly, primarily in length. RNase protection assays that use RNA isolated from specific $S G$ regions show that SqKv1A mRNA is expressed prominently in the GFL but not in the SG proper. SqKv1B yields the opposite pattern. SqKv1D also is expressed only in the SG. SqKv1C expression was not detectable. In situ hybridizations confirm these results and reveal that SqKv1B mRNA is abundant in many large neurons of the SG, whereas SqKv1D expression is limited to small isolated clusters of neurons. SqKv1A and B are thus the predominant Kv1 mRNAs in the SG/GFL complex. Activation properties of SqKv1A and B channels expressed in oocytes are very similar to one another and compare favorably with properties of native delayed rectifier channels in GFL neurons and large SG neurons. The Kv1 complement in these squid neurons thus seems to be relatively simple. Several differences exist between cloned and native channels, however, and may reflect differences in the cellular environments of oocytes and neurons.

Key words: squid giant axon; cloning; Kv1 genes; potassium channels; expression; alternative splicing
Squid giant axons are nonmyelinated motor fibers, up to $500 \mu \mathrm{m}$ or more in diameter, that innervate circular muscle fibers of the mantle, and a single axonal action potential excites the entire motor field in an all-or-none manner (Young, 1938). These axons are formed by fusion of hundreds of very small axons, only several micrometers in diameter, originating from monotypic giant fiber lobe (GFL) neurons localized to the posterior tip of the stellate ganglion (SG) (Young, 1939). A second, less well characterized motor system consists of "ordinary" small diameter axons (Prosser and Young, 1937) that arise from large cell bodies, up to $100 \mu \mathrm{m}$ in diameter, in the SG proper (Young, 1972). Repetitive activity in this small axon system produces graded excitation of circular muscle fibers (Wilson, 1960; Gilly et al., 1996), and during escape jetting the giant and small axon systems are recruited in concert (Otis and Gilly, 1990).

A major focus of research on the giant axon system has been the $\mathrm{Na}$ and $\mathrm{K}$ channels underlying action potential transmission, and recent physiological and molecular approaches have converged on the delayed rectifier $\mathrm{K}$ conductance $\left(g_{\mathrm{K}}\right)$. A K channel with a unitary conductance of $20 \mathrm{pS}$ makes up the delayed rectifier $g_{\mathrm{K}}$ in both cell bodies (Llano and Bookman, 1986; Nealey et al., 1993) and giant axons (Perozo et al., 1991), and a cDNA, SqKv1A, has been postulated to correspond to the mRNA encoding this channel (Rosenthal et al., 1996). Other K channel types exist in giant

Received Feb. 7, 1997; revised April 16, 1997; accepted April 21, 1997.

This work was supported by National Institutes of Health Grant NS17510 to W.G., an Office of Naval Research Augmentation Award for Science and Engineering Research Training to J.R., and an individual United States Public Health Service award to M. Perri, who contributed to several aspects of this project.

Correspondence should be addressed to Dr. W. F. Gilly at the above address.

Dr. Rosenthal's present address: Department of Physiology, University of California at Los Angeles Medical Center, Los Angeles, CA 90095.

Copyright (C) 1997 Society for Neuroscience $0270-6474 / 97 / 175070-10 \$ 05.00 / 0$ axons (Llano et al., 1988) and GFL cell bodies (Nealey et al., 1993), but they contribute a comparatively minor fraction of macroscopic $g_{\mathrm{K}}$.

In the course of characterizing SqKv1A expression, in situ hybridizations suggested that, within the SG, mRNA for this channel was expressed selectively in GFL neurons. This degree of specificity seemed surprising, because the proposed functional role for this channel in action potential transmission could hardly be specific to the giant axon. If SqKv1A mRNA encodes delayed rectifier $\mathrm{K}$ channels for the giant axon system, what kind of $\mathrm{K}$ channels are present in the axons and cell bodies of the functionally similar small motor axon system?

This paper describes four closely related mRNAs, at least three of which are expressed in neurons of the SG/GFL complex. All four cDNAs encode Kv1 channels with predicted structures nearly identical to that of SqKv1A. Only SqKv1A, however, appears to be expressed in GFL neurons. One of the new mRNAs, SqKv1B, is expressed widely in cell bodies distributed in the SG proper, including the largest cells that constitute the somata of the small axon motor system. Functional properties of native $g_{\mathrm{K}}$ in GFL neurons and large SG neurons are generally comparable to one another and to properties of SqKv1A and B expressed in oocytes.

\section{MATERIALS AND METHODS}

Library screening. Screening of a SG/GFL cDNA library $(\lambda$-UNI-ZAP XR; Rosenthal and Gilly, 1993) was performed as described elsewhere (Rosenthal et al., 1996). Briefly, degenerate primers NEYFFD and FWWAVV were used to amplify an 858 base pair (bp) fragment of a Kv1 cDNA (pSKC1; see Fig. 1) from GFL mRNA by PCR. ${ }^{32}$ P-labeled, random-primed probe (Sambrook et al., 1989) was made from pSKC1 template and used to screen $\sim 10^{6}$ plaques.

Size selection of positive plaques. DNA fragments encoding $5^{\prime}$ ends of Kv1-type channels were amplified from individual positive plaques by 


\begin{tabular}{lll}
\hline \multicolumn{2}{l}{ Table 1. Oligonucleotides used for PCR amplifications, sequencing, Southern blots, and cloning procedures } \\
Name & Sequence & Location \\
\hline T3 & AATTAACCCTCACTAAAGGG & pBluescript \\
T7 & AATACGACTCACTATAG & pBluescript \\
SKC4 & GTGCCAAATTTTGGCATGG & $629 \rightarrow 647$ of SqKv1A \\
SKC7 & AGCGACGTGTATGGCTCTTG & $458 \rightarrow 478$ of SqKv1A \\
SKC8 & CAACGTGATGTTTAGAAAAG & $-7 \rightarrow 13$ of SqKv1A \\
SKC9 & ACTAGGACGATTCCCATC & $256 \rightarrow 273$ of SqKv1A \\
SKC14 & TCTGTGGATCCAATCTTGCCTCCTAC & $-212 \rightarrow-186$ of SqKv1C \\
SKC16 & CATAAGATCTCAGACATCGGTTTC & $1452 \rightarrow 1469$ of SqKv1A \\
SKC20 & GCAAAGATCTTCTGCCATGTCTCTTGAC & $-16 \rightarrow 11$ of SqKv1B-pBSTA \\
SKC21 & TTTAAACTCTCGCGCCATTCC & $895 \rightarrow 915$ of SqKv1A \\
SKC22 & TGTGGCAGAAGAGACTGTTG & $1273 \rightarrow 1293$ of SqKv1A \\
SKC30 & TCAGGGATCCCAATGGCAATTACGTTG & $-155 \rightarrow-139$ of SqKv1A \\
SKC31 & GCCGAAGCTTCTAAACATCACGTTGTTCC & $-11 \rightarrow 10$ of SqKv1A \\
SKC32 & TCCGAAGCTTTGGGAGAATATCCTTGCG & $-17 \rightarrow 3$ of SqKv1B \\
SKC33 & TATCAAGCTTCATTTCCGGCCTGGAGGTC & $-16 \rightarrow 3$ of SqKv1C \\
SKC34 & TCCGAAGCTTTCGTCAGACTCCCTTAGTG & $-26 \rightarrow-7$ of SqKv1D
\end{tabular}

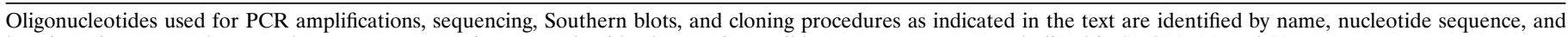
location of correspondence to the vector or cDNA insert. Nucleotide changes from wild-type sequences are underlined in SKC14, 16 , and 20.

PCR, using the T3/SKC4 primer pair. Each reaction contained $5 \mu$ l of boiled supernatant from the plaque stock [cored primary plaque in sodium-magnesium (SM) buffer $\left(100 \mathrm{~mm} \mathrm{NaCl}, 8 \mathrm{~mm} \mathrm{MgCl}_{2}, 50 \mathrm{~mm}\right.$ Tris, $\mathrm{pH} 7.5$, and $0.01 \%$ gelatin)], $5 \mu \mathrm{l}$ of $10 \times \mathrm{Taq}$ polymerase buffer $(100$ mu Tris, $\mathrm{pH} 8.3,12.5 \mathrm{~mm} \mathrm{MgCl}_{2}, 500 \mathrm{~mm} \mathrm{KCl}$, and $0.1 \%$ of both NP-40 and Triton X-100) containing $1 \mu \mathrm{M}$ of each primer and $1 \mathrm{U}$ of Taq polymerase. Reactions were performed in a DNA thermocycler (PerkinElmer, Branchurg, NJ) and consisted of 30 cycles of denaturation $(30 \mathrm{sec}$, $92^{\circ} \mathrm{C}$ ) and elongation $\left(120 \mathrm{sec}, 72^{\circ} \mathrm{C}\right)$. Annealing ( $60 \mathrm{sec}$ per cycle) was at $60^{\circ} \mathrm{C}$ for the first five cycles and at $50^{\circ} \mathrm{C}$ thereafter.

Sizes of PCR products were checked on $1 \%$ agarose gels, and only plaques yielding PCR products $>600 \mathrm{bp}$ were analyzed further. These gels were dried and used as a matrix for hybridization with ${ }^{32} \mathrm{P}$ endlabeled oligonucleotides SKC 8 and SKC7 in $6 \times$ SSC $(150 \mathrm{~mm} \mathrm{NaCl}$ and $15 \mathrm{~mm}$ Na-citrate, $\mathrm{pH} 7$ ) plus $250 \mu \mathrm{g} / \mathrm{ml}$ sonicated calf thymus DNA. Gels were hybridized with $3 \times 10^{6} \mathrm{cpm}$ probe $/ \mathrm{ml}$ at $55^{\circ} \mathrm{C}$ for $16 \mathrm{hr}$, washed in $6 \times \mathrm{SSC}$ and $0.1 \%$ SDS for $3 \times 30 \mathrm{~min}$ at $58^{\circ} \mathrm{C}$, dried again, and exposed to $\mathrm{x}$-ray film.

$D N A$ sequencing. Plasmids containing clones of interest were excised in vivo (Rosenthal and Gilly, 1993). Sequencing of SqKv1A is described elsewhere (Rosenthal et al., 1996). SqKv1B also was sequenced in both directions by constructing nested deletions. SqKv1C and D were sequenced by using primers derived from SqKv1A and B sequence. PCR products were sequenced directly by unidirectional PCR with ${ }^{33} \mathrm{P}$-ATP end-labeled primers, using the fMol system (Promega, Madison, WI). All differences among SqKv1A-D described here were verified by conventional dideoxy sequencing of the $5^{\prime}$ untranslated region (UTR) and coding regions of randomly selected representative clones of each type.

Tissue preparation and RNA isolation. Loligo opalescens was collected from Monterey Bay, CA. Squid were decapitated, and complete SG/GFLs (i.e., SG with GFL attached) were removed immediately and dissected into three pieces consisting of GFL tissue, the anterior portion of the SG proper (simply referred to as SG), and the zone between these regions. This provided highly enriched samples of GFL neurons and SG neurons used for RNA extraction and a mixed population that was discarded. Tissue samples were frozen in liquid nitrogen and processed for RNA extraction (Rosenthal and Gilly, 1993).

RNase protection assays. So that templates for SqKv1A-D probe synthesis could be created, full-length cDNAs (cloned into the EcoRI and XhoI sites of pBluescript $\mathrm{SK}^{-}$) were digested with $\mathrm{XhoI}$ and NdeI, blunt termini were created with Klenow fragment, and the plasmids were recircularized with T4 DNA ligase. For in vitro transcription of cRNA probes, the SqKv1A-derived plasmid was linearized with EagI in the 5' UTR (see Fig. 1), and SqKv1B-D were linearized with Bam HI in the 5' polylinker. Because the 57 nucleotides (nt) upstream from the NdeI site (nt 101 in SqKv1A, 68 in SqKv1B, and -2 in SqKv1D) are identical in SqKv1A-D, it was hoped that protection of this region by nonhomologous probes could serve as an internal control in these experiments.
Protection of this fragment proved to be variable, however, for unknown reasons.

${ }^{32}$ P-labeled cRNA probes were synthesized with T7 RNA polymerase (Riboprobe kit, Promega) and used for RNase protection assays as previously described (Rosenthal and Gilly, 1993; Rosenthal et al., 1996). Briefly, $5 \mu \mathrm{g}$ aliquots of GFL or SG total RNA were hybridized with $10^{6}$ $\mathrm{cpm}$ of probe for $16 \mathrm{hr}$ at $48^{\circ} \mathrm{C}$. Each probe also was hybridized with 10 $\mu \mathrm{g}$ of tRNA as a control, and $10^{3} \mathrm{cpm}$ of nondigested probe provided a size marker.

In situ hybridizations. Complete SG/GFLs were fixed in cold paraformaldehyde, processed, and frozen as previously described (Liu and Gilly, 1995). Frozen sections $(12-15 \mu \mathrm{m})$ were cut longitudinally in the plane parallel to the dorsal surface of the mantle, melted onto silane-coated slides (Polysciences, Warrington, PA), and stained with cresyl violet. Slides were washed, dehydrated in graded ethanol, and desiccated before use in hybridizations.

Probe templates specific for SqKv1A-D, used to synthesize ${ }^{35}$ S-labeled antisense cRNA, were constructed by PCR amplification of unique regions of the $5^{\prime}$ end of each cDNA with the following sense/antisense primers (Table 1): SK30/SKC31 for SqKv1A, T3/SKC32 for SqKv1B, T3/SKC33 for SqKv1C, and T3/SKC34 for SqKv1D. Products were subcloned into pBluescript $\mathrm{SK}^{-}$. These probes differ from those used for RNase protection assays in that they contain no sequence shared by the four SqKv1 cDNAs. A ${ }^{35}$ S-labeled sense cRNA probe to control for nonspecific hybridization was transcribed with T3 RNA polymerase from a squid Na channel template (pNC5'; Rosenthal and Gilly, 1993). All probes were used at a final working concentration of $5 \times 10^{6} \mathrm{cpm} / \mathrm{ml}$. Protocols for probe synthesis and in situ hybridization are described elsewhere (Simmons et al., 1989; Liu and Gilly, 1995).

Constructs for functional expression. A plasmid for synthesis of fulllength SqKv1A cRNA (SqKv1A-pBSTA) was constructed with the pBSTA vector (previously called Chi 7; Rosenthal et al., 1996) that contains $5^{\prime}$ and $3^{\prime}$ Xenopus $\beta$-globin UTR. An analogous SqKv1BpBSTA construct was created as follows. BglII sites were introduced adjacent to the start and stop codons of SqKv1B by PCR amplification of the complete coding region by using primers SKC20 and SKC16 (Table 1). SKC20 contains a consensus translation initiation sequence (Kozak, 1989), and incorporation of this feature resulted in changing the second amino acid of SqKv1B from the wild-type Ser to Ala. To avoid errors introduced by Taq DNA polymerase, we replaced the NdeI-SpeI fragment of SqKv1B-pBSTA (nt 68-1113; see Fig. 1), containing most of the $\mathrm{SqKv1B}$ coding region, with the same region from the original SqKv1B cDNA. Regions upstream of the NdeI site and downstream of the SpeI site were resequenced.

Oocyte preparation and patch-clamp recording. Stage IV Xenopus oocytes were treated with $1.5 \mathrm{mg} / \mathrm{ml}$ collagenase Type IA (Boehringer Mannheim, Indianapolis, IN) in Ca-free saline containing (in $\mathrm{mM}$ ): 82 $\mathrm{NaCl}, 2 \mathrm{KCl}, 1 \mathrm{MgCl}_{2}$, and $5 \mathrm{HEPES}, \mathrm{pH} 7.6$, before injection with $\sim 10$ 
ng of cRNA synthesized from $5 \mu \mathrm{g}$ of SqKv1A- and SqKv1B-pBSTA plasmid (linearized with NotI) using a kit (Message Machine version 9055, Ambion, Austin, TX). Injected oocytes were incubated in ND96 medium containing (in $\mathrm{mM}$ ): $96 \mathrm{NaCl}, 2 \mathrm{KCl}, 1.8 \mathrm{CaCl}_{2}, 1 \mathrm{MgCl}_{2}$, and 5 HEPES plus penicillin $\left(10^{4} \mathrm{U} / \mathrm{ml}\right)$, streptomycin $(10 \mathrm{mg} / \mathrm{ml})$, and $2.5 \mathrm{~mm}$ Na-pyruvate at $17^{\circ} \mathrm{C}$ for $2-4 \mathrm{~d}$. Immediately before recordings were made, an oocyte was bathed in a hypertonic solution containing (in $\mathrm{mM}$ ): $200 \mathrm{NMG}$-aspartate, $20 \mathrm{KCl}, 10$ EGTA, $1 \mathrm{MgCl}_{2}$, and $10 \mathrm{HEPES}, \mathrm{pH} 7.4$, for 5-10 min, and the vitelline membrane was removed manually.

Cell-attached patch recordings were made at $\sim 18^{\circ} \mathrm{C}$. Pipettes $(1-2$ $\mathrm{M} \Omega$ ) were filled with (in $\mathrm{mm}$ ): $118-90 \mathrm{NaCl}, 2-30 \mathrm{KCl}, 6 \mathrm{MgCl}_{2}$, and 5-10 HEPES, $\mathrm{pH}$ 7.2. $\mathrm{NaCl}$ was replaced with $\mathrm{KCl}$ on an equimolar basis for the bath solution. The voltage-clamp and data acquisition system were conventional (Rosenthal et al., 1996). Signals were filtered at 5 or $0.5 \mathrm{kHz}$ with an 8-pole Bessel filter before sampling at $20-50$ or $2 \mathrm{kHz}$, respectively.

Recordings from squid neurons. Complete SG/GFLs were dissected as described above into GFL and SG portions, which then were treated with nonspecific protease $(7 \mathrm{mg} / \mathrm{ml}$ Type XIV, Sigma, St. Louis, MO) for 50 min (room temperature) to isolate cell bodies for short-term culture (Gilly et al., 1990). Cell-attached patch recordings were performed at $\sim 18^{\circ} \mathrm{C}$ within $1-2 \mathrm{~d}$ of isolation. The bath solution contained (in $\mathrm{mM}$ ): 115 $\mathrm{NaCl}, 385 \mathrm{KCl}, 10 \mathrm{MgCl}_{2}, 10 \mathrm{CaCl}_{2}$, and 10 HEPES, pH 7.5 (980 mOsm). Pipettes were filled with (in mM): $400 \mathrm{NaCl}, 100 \mathrm{KCl}, 10 \mathrm{MgCl}_{2}, 10$ $\mathrm{CaCl}_{2}, 10$ HEPES, and 0.0005 TTX, pH 7.5 (980 mOsm).

Conventional whole-cell recordings were performed on dissociated GFL neurons as described in detail elsewhere (Mathes et al., 1997). The external solution, designed to approximate the ionic strength and composition of the pipette solution for oocyte recordings, contained (in $\mathrm{mM}$ ): $75 \mathrm{NaCl}, 20 \mathrm{KCl}, 20 \mathrm{MgCl}_{2}, 5 \mathrm{CaCl}_{2}, 10 \mathrm{HEPES}$, and $0.003 \mathrm{TTX}, \mathrm{pH} 7.5$, plus sucrose to attain $980 \mathrm{mOsm}$. The internal (pipette) solution for whole-cell recordings was also of low ionic strength and contained (in $\mathrm{mM}$ ): $25 \mathrm{KF}, 25 \mathrm{KCl}, 50 \mathrm{~K}$-glutamate, $10 \mathrm{~K}_{2}$-EGTA, $\mathrm{pH} 7.4$, and sucrose to attain $980 \mathrm{mOsm}$.

\section{RESULTS}

\section{Identification of squid Kv1 cDNAs}

Our initial screen of the SG/GFL cDNA library used a probe derived from a partial squid Kv1 cDNA that was isolated by PCR from GFL mRNA (pSKC1; Rosenthal et al., 1996). Sixty-nine positive clones were identified, and the corresponding cored phage plaques were tested with PCR by using a sense primer to the cloning vector (T3, Table 1) and an antisense primer specific to sequence encoding the intracellular linker between transmembrane segments S1 and S2 of SqKv1A (SKC4, Fig. 1 $A$, Table 1). Eleven of these reactions failed to amplify, and PCR analysis with primers $\mathrm{T} 3$ and $\mathrm{T} 7$ revealed that the inserts were significantly smaller than the full-length coding region of a typical Kv1 cDNA. These clones were not analyzed further. Of the 58 successfully amplified products, 30 were $\geq 600 \mathrm{bp}$ in size and therefore probably sufficiently large to include the complete $\mathrm{N}$-terminal coding region. The clone yielding the largest PCR product contained SqKv1A, a full-length cDNA encoding a squid Kv1 channel (Rosenthal et al., 1996). The remaining 29 clones were analyzed as described below.

\section{A family of SG/GFL Kv1 cDNAs based on 5' diversity}

$\mathrm{N}$ termini of $\mathrm{Kv} 1 \alpha$-subunits, even closely related ones in the same species, are highly divergent. This can be attributable to alternative mRNA splicing, as occurs with the Shaker locus in Drosophila (see also Discussion; Papazian et al., 1987), or to the existence of different genes, as seen in mammalian RCK1-5 [Stuhmer et al. (1989); see also Jegla et al. (1995)]. We therefore analyzed $\mathrm{N}$-terminal diversity in our 30 potentially full-length Kv1 cDNAs. An oligonucleotide probe spanning the assigned translation start site of SqKv1A (SKC8; Fig. 1 $A$, Table 1) was hybridized under stringent conditions to a Southern blot of the PCR products encoding the $5^{\prime}$ ends of these 30 clones. Only 19 samples yielded positive hybridization signals, whereas hybridization with another oligonucleotide (SKC7, Fig. $1 A$, Table 1 ) that encodes a region of SqKv1A highly conserved among all Kv1 channels (Drewe et al., 1990; Li et al., 1992) occurred with all 30. Direct sequencing of the 19 SKC8-positive PCR products that used T3 and SKC9 primers (Fig. $1 A$, Table 1 ) established that these cDNAs were identical to SqKv1A in this region.

Sequencing of the $11 \mathrm{PCR}$ products that failed to hybridize to SKC8 revealed three additional species of Kv1 cDNAs. The nomenclature of these cDNAs and their frequency of occurrence in the described set of 30 clones are SqKv1A $(n=19)$, SqKv1B $(n=9), \operatorname{SqKv} 1 \mathrm{C}(n=1)$, and SqKv1D $(n=1)$. A representative of each type was sequenced to completion, and nucleotide sequences for SqKv1A-D are given in Figure $1 B$. Each squid Kv1 cDNA has a unique $5^{\prime}$ end corresponding to sequence upstream from nt 35 of SqKv1A. Sequence downstream of this site is highly homologous in all four cDNAs but does exhibit sporadic differences (10 total) that are all A-G conversions, with the exception of the position corresponding to nt 35 of SqKv1A.

\section{Examination of homology at the $3^{\prime}$ end of SqKv1A-D cDNAs}

All 30 full-length cDNAs discussed above also were tested for 3' diversity. PCR primers SKC21 and SKC22 (Fig. $1 A$, Table 1) were used to amplify the coding region flanking and including the S5 and S6 transmembrane regions of SqKv1A (nt 915-1292). This region includes a short (24 nt) stretch of DNA that is highly divergent in closely related rat Kv1 cDNAs (Stuhmer et al., 1989) and encodes part of the pore-forming region (Jan and Jan, 1992; Pongs, 1992). Direct sequencing of the 30 amplified fragments revealed no variability, however. Similarly, analysis of the C-terminal coding region and $3^{\prime}$ UTR by direct sequencing of PCR products obtained with T7 and SKC21 primers showed no variability. Although SqKv1A-D cDNAs have distinct 5' UTR and $\mathrm{N}$-terminal coding sequence, they share $\mathrm{C}$-terminal coding and a 3' UTR sequence.

\section{Comparison of primary structures}

The predicted amino acid sequence of SqKv1A has been compared with that of Shaker B elsewhere (Rosenthal et al., 1996). None of the SqKv1 cDNAs includes a well defined translation initiation sequence (Kozak, 1989), and putative start sites in each case (Fig. $1 B$ ) were assigned to the methionine codon that yielded the longest open reading frame. Differences in the locations of the start sites and scattered base differences in SqKv1A-D lead to predicted $\mathrm{N}$ termini that differ in length and composition (Fig. $2 A$ ). After the position equivalent to $\mathrm{G} 48$ of SqKv1A (diamonds in Fig. 2), SqKv1A-D differ at only four residues, as indicated in Figure 2B. Overall identities between SqKv1A and SqKv1B, C, and $\mathrm{D}$ are 96,97 , and $92 \%$, respectively.

Although variability exists in the $\mathrm{N}$ termini of SqKv1A-D, the functional significance of this is unknown. SqKv1A begins with a unique stretch of 12 amino acids, and residues 5-10 are unusually hydrophobic, as revealed by Kyte-Doolitte hydropathy analysis of a number of Kv1 $\alpha$-subunits (data not shown). Although this structure has the motif of a conventional leader peptide (Von Heinje, 1990; Dalbey and Von Heinje, 1992), it is probably too short to function in this capacity. SqKv1B and C both lack this hydrophobic $\mathrm{N}$ terminal and are nearly identical to one another. SqKv1D has the shortest $\mathrm{N}$ terminus, lacking the first 34 residues of SqKv1A.

None of the predicted differences among SqKv1A-D occurs 
A

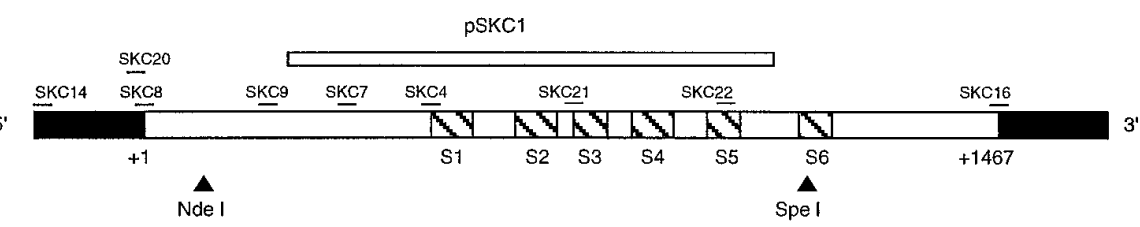

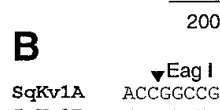

$200 \mathrm{bp}$

SqKv1B

SqKv1C

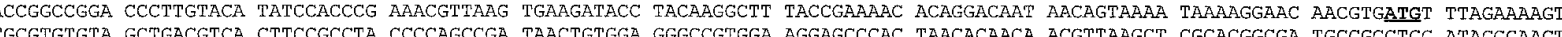
GTA GCTGACGTCA CTTCCGCCTA CCCCAGCCGA TAACTGTGGA GGGCCGTGGA AGGAGCCCAC TAACACAACA ACGTTAAGCT CGCACGGCGA TGCCGCCTGG ATACGGAACT

SqKviD ACTGCCTCCT TTTCTATATA TGTCTTTCAT TCGTTCTTCC TCTCAATATT GCTACAAGTC TACGCGCTCC CTAGTCCTAT GTTAAGGGAC GGACGGGTTG GATGTCTGCA GAAATATTTA
CCTAGTCC TATGTTAAGG GACGGACGGG TTGGATGTCT GCAGAAATAT TTAGACCTCC AGGCCGGAAA TGAGAAGGCA GTCACACTAA

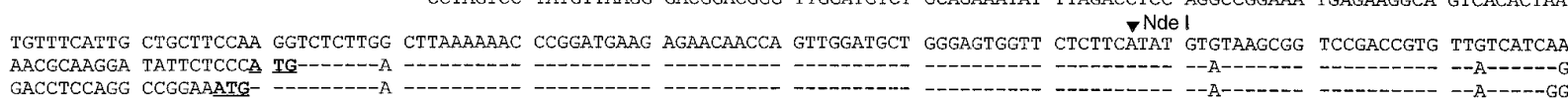
GACCTCCAGG CCGGAAATG CGTAAGCGGA CTTCGGTITG AAACACAAAC CCGTACACTA AGCCAGTTTC CGGATACGTT GTTGGGTAAC CCCAAAAAGC GAAACCGCTA TTATGACCCA TACCGGARCG AATATTTTTT ----G---- - CGGGGAGGAG GTCATTGACA AATACCGTGC AGAAGAGGGA TTTGTCAAGG AAGTAGAAAA ACCCCTTCCC GAGAACGAT TCCAGCGACG TGTATGGCTC TTGGTTGAGC ACCCGGAGAG С TTCTACTCCG GCCCGGGTGA TCGCTATAGT GTCCGTTAGC GTCATTGTGA TCTCAATAGT TACCTTCTGC TTAGAAACTC TGCCAGAGTT CAAACGATAT GAACTTCTGA ACGA -

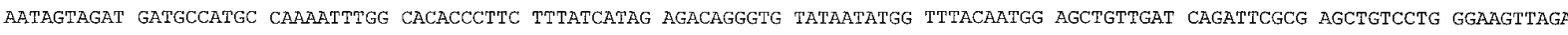
TTTCTTTAAG GACAGCATGA ACGCTATCGA TGTTGTGTCG ATTATGCCGT ACTTCATAAC ACTGGGAACC GTAATCCAAA ACGATACGAA CACCCAAGAT AAACAGAATA TGTCGCTIGC - AATCCTCAGA GTAATTCGTC TAGTCAGAGT GTTTCGAATA TTTAAACTCT CGCGCCATTC CAAAGGTCTG CAAATCTTG GCCAGACACT GAAAGCTAGT TTACGCGAGC TCGGTTTGCT CATTTTCTTT CTTGTGATTG GTGTTGTCTT ATTTTCCAGC GCCGTCTACT TCGCAGAAGT GGACACGGAA CAGAGTCATT TCAAAGCAT TCCTGACTCA TTTTGGTGGG CCGTTGTAAC -

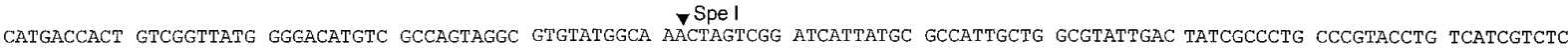
С-_AAATTTCAAT TACTTTTACC ACCGAGACGC AGAAGCTACI GGCGACAAGg AAAAGTTCCA ACATGTCTCT TCTGCCACAA ATTATTACGA CGAGAAAATC AGTTCGTATA CGTCGGAAGT АAA TGAGTCTGAC ATTATGGAAA TGGACGAAGg AAGCATGTAT ACTGACAAGT GGAAAGAAGg AAATCTTAGT AACACCAACA ATATTGGGTT TAATCACATC AAAAATAACC TCAGTATGCA

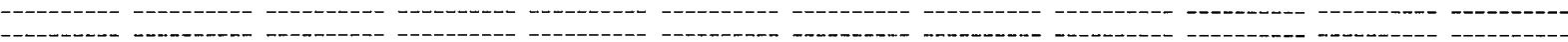
-

AACCGATGTC TGP̈GAGACTA TGGCCTTGAT ACATGCAAGA TACCAAAAAG GAAAAAAAA AGCGAAAGA AGAAAAGAAA AAAAAATAAA CGAARTTTTA AACAAATTTT AAAAAAAAAA

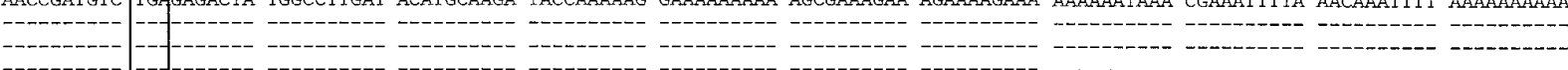
AAAAAAAAA 1583 AAAAAAAAA $(-0-10$

Figure 1. Structure of the SqKv1 cDNAs. A, Schematic of SqKv1A coding and UTRs (open and solid segments, respectively) showing the locations of oligonucleotides used in this study (see Table 1) and of the probe ( $p S K C 1$; Rosenthal et al., 1996) used to screen the cDNA library. Cross-hatched segments represent transmembrane regions S1-S6. The restriction sites indicated are discussed in Materials and Methods and Results. B, Nucleotide sequences of SqKv1A-D cDNAs. Numbering pertains to SqKv1A. Identities to SqKv1A are indicated by dashes. Start codons (boldface and underlined) were assigned as the first Met codon that yielded the longest open reading frame. Stop codons are boxed. The four nucleotide sequences become virtually identical downstream from position 35 of SqKv1A. The locations of the NdeI and EagI sites discussed in Materials and Methods and Results are indicated for $S q K v 1 A$. The complete 5' UTR sequence is given for $S q K v 1 D$; the others have been truncated arbitrarily. Complete sequences will be deposited in GenBank on acceptance of this manuscript. 
A

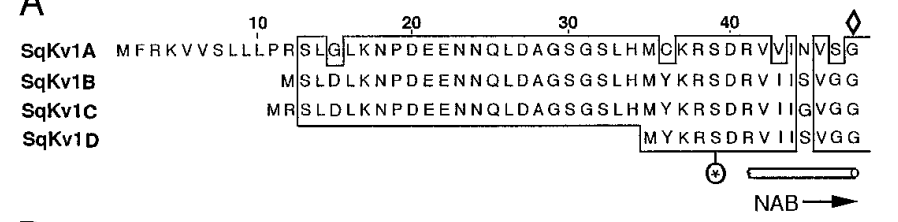

B

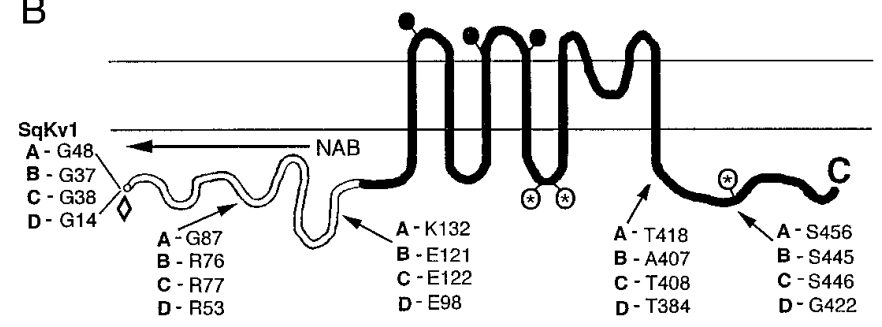

Figure 2. Differences in predicted primary structure of squid Kv1 $\alpha$-subunits, based on SqKv1A-D cDNAs. $A$, Alignment of the predicted $\mathrm{N}$-terminal portions of SqKv1A-D. Identical residues are boxed. Illustrated sequences stop at the position equivalent to $\mathrm{G} 48$ (diamond) of $\mathrm{SqKv} 1 \mathrm{~A}$ because all four of the predicted SqKv1 channels are identical after this point, with the exception of the four sites noted in $B$. The complete sequence of SqKv1A is given elsewhere (Rosenthal et al., 1996). A consensus intracellular PKC site (asterisk) is indicated near the start of the NAB domain (indicated by white bar below the sequences). $B$, Schematic of the SqKv1A protein between G48 (diamond) and the C terminus. The four residues downstream from G48 that differ in SqKv1A-D are noted, and their approximate locations are indicated. The white segment of the channel corresponds to the continuation of the NAB domain. Consensus intracellular PKC sites (asterisks) and extracellular N-linked glycosylation sites ( filled circles) are indicated. SqKv1A-D show no consensus PKA sites.

within a putative membrane-spanning, pore-forming, or intracellular linker region (Jan and Jan, 1992; Pongs, 1992). Potentially significant differences do exist, however, in the NAB domain (Drewe et al., 1992), the cytoplasmic N-terminal region known to mediate both $\alpha-\alpha$ (Li et al., 1992; Shen and Pfaffinger, 1995; Xu et al., 1995) and $\alpha-\beta$ (Yu et al., 1996; Sewing et al., 1996) subunit interactions. Positions corresponding to S47 and G87 of SqKv1A are replaced by Gly and Arg, respectively, in SqKv1B-D. A Ser at the equivalent $\mathrm{S} 47$ position seems to be universally conserved among Kv1-4 $\alpha$-subunits, based on alignments of $44 \mathrm{Kv} 1,7 \mathrm{Kv} 2$, $10 \mathrm{Kv} 3$, and $5 \mathrm{Kv} 4$ members provided by Dr. M. Li (Johns Hopkins University, Baltimore, MD), except for SqKv1B-D. SqKv1A, however, seems to be unique in having a Gly at the position equivalent to G87. In the same $66 \mathrm{Kv} \alpha$-subunits, as well as in SqKv1B-D, an Arg appears at this position, and the six residues immediately upstream of this site (NEYFFD) are also absolutely conserved. A third difference in the NAB domain occurs at K132 of SqKv1A, which is replaced by a Glu in SqKv1B-D. A basic residue at this position is found in many, but not all, Kv1 $\alpha$-subunits. The significance of these anomalous residues in the $\mathrm{SqKv} 1$ members is unclear.

\section{Specificity of expression of SqKv1A-D mRNAs within the stellate ganglion}

RNase protection assays

A series of antisense cRNA probes was used to detect the unique $5^{\prime}$ ends of SqKv1A-D mRNAs and thereby determine their distribution patterns in the SG/GFL complex. Only results for SqKv1A, B, and D are illustrated here; results with SqKv1C probe have been inconclusive because of nonspecific hybridization in control tests with tRNA. ${ }^{32} \mathrm{P}$-labeled probes were used in separate RNase protection assays with RNA isolated from either GFL or

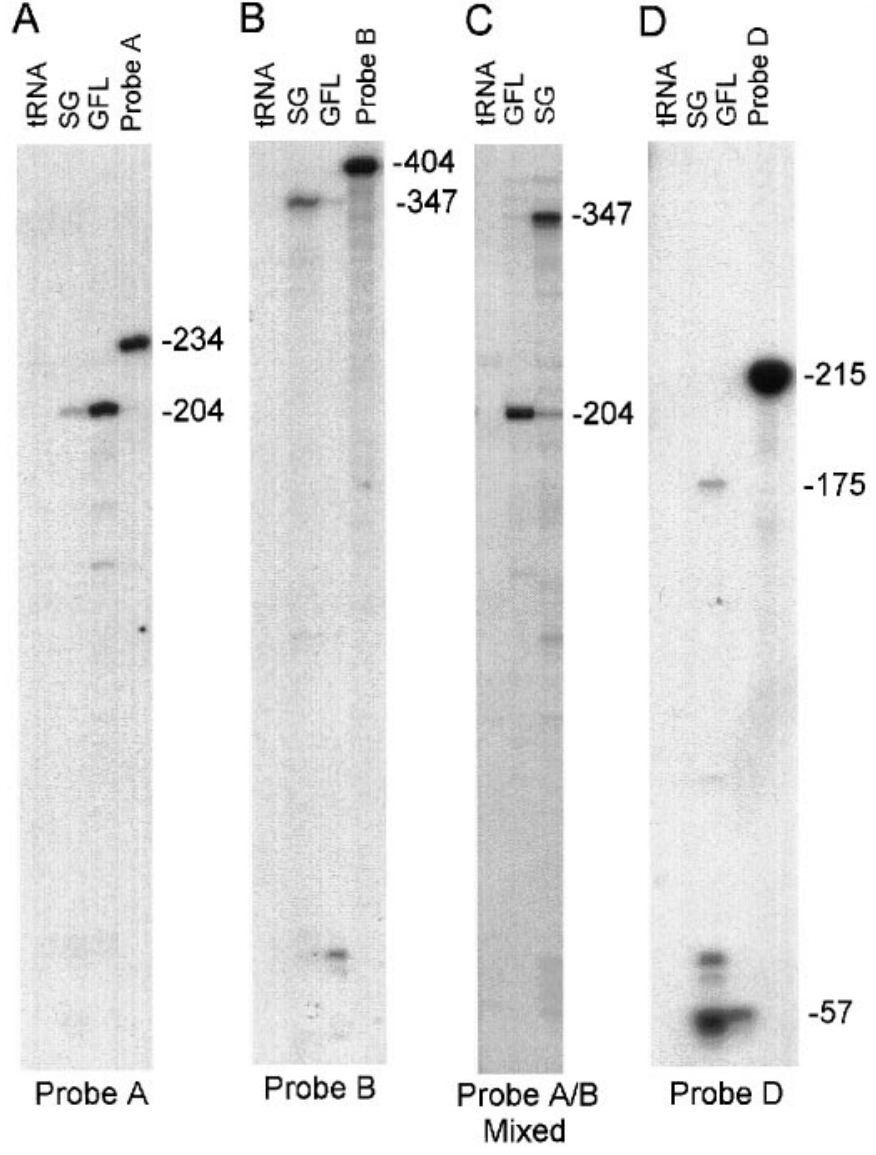

Figure 3. Expression of SqKv1A, B, and D mRNAs in the SG/GFL complex, as determined by RNase protection assays. Predicted sizes for undigested probes and fully protected bands are (in nt) 234/204 (SqKv1A), 404/347 (SqKv1B), and 215/175 (SqKv1D). A, RNase protection assays were performed with SqKv1A probe (Probe $A$ ) and RNA selectively isolated from either SG and GFL, as indicated at the top of each lane. $G F L$, Giant fiber lobe; $S G$, anterior portion of stellate ganglion (see Materials and Methods). $t R N A$ served as a negative control. Nondigested probe provided a size marker. A fully protected band is prominent in the GFL lane (7 d exposure). B, RNase protection assays were performed with SqKv1B probe (Probe B) in the manner described. A prominent, fully protected band is evident in the $S G$ lane ( $7 \mathrm{~d}$ exposure). $C$, Results of another experiment in which assays were performed with both SqKv1A and B probes (Probe A/B Mixed). A 204 nt band corresponding to SqKv1A mRNA is very prominent in the GFL lane; a $347 \mathrm{nt}$ band corresponding to $\mathrm{SqKv} 1 \mathrm{~B}$ is apparent in the $S G$ lane. $D$, RNase protection assays performed with SqKv1D probe (Probe $D)$ yield a clear band only in the $S G$ lane $(1 \mathrm{~d}$ exposure; same experimental series as $A, B)$. See text for additional details.

anterior SG samples (see Materials and Methods), and results are shown in Figure $3 A-D$. SqKv1A probe is predicted to produce a fully protected band of $204 \mathrm{nt}$ with its complementary RNA, and a band of this size (determined by a parallel assay with SqKv1A cRNA; not illustrated) is obvious in the GFL lane of Figure $3 \mathrm{~A}$. Only a very weak band exists in the SG lane. Conversely, in Figure $3 B$ SqKv1B probe yields a strong, protected band of $347 \mathrm{nt}$ only with SG RNA. This segregated pattern of distribution also is demonstrated by another experiment in which both probes were applied simultaneously to GFL and SG RNA samples (Fig. 3C).

These results confirm that SqKv1A mRNA is much more abundant in GFL neurons than in SG neurons (Rosenthal et al., 1996) and show that the opposite is true for SqKv1B mRNA. SqKv1D mRNA also is expressed in the SG and not in the GFL, and this 
is shown by results of an assay with SqKv1D probe (Fig. 3D) in which a fully protected band of $175 \mathrm{nt}$ is detectable only in the SG sample. The level of expression for SqKv1D relative to that of SqKv1B (or A) cannot be ascertained from these experiments alone, because specific activities of the probes are not known. Based on the intensity of the nondigested probe signal in Figure $3 D$, the specific activity of probe $\mathrm{D}$ in this experiment would seem to be anomalously high. This, however, permits detection of the smaller protected fragments predicted to be $\sim 57$ bp in both SG and GFL samples because of probe sequence that is common to SqKv1A, B, and D (see Materials and Methods and Fig. 1B). The presence of these bands is consistent with an abundant amount of SqKv1B mRNA in the SG sample and shows that spurious RNase contamination of the GFL sample was not responsible for the negative result with the $\mathrm{SqKv} 1 \mathrm{D}$ probe.

\section{In situ hybridizations}

In situ hybridization was used to map the distributions of SqKv1A-D mRNAs in the SG/GFL complex more. Figure $4 A i$ shows a stained section in which the GFL comprises the left portion of the ganglion, and tracts of fusing axons arising from GFL neurons (arrowheads) project toward the central neuropil of the SG proper. Portions of stellar nerves are visible, and giant axons in two of them are denoted by asterisks. The border between the GFL neurons and the much larger neurons of the SG is quite distinct (Fig. 4Aii).

SqKv1A probe selectively labeled GFL neurons (Fig. 4Bi,Bii), whereas the SqKv1B-specific probe identified many large neurons in the SG but very few, if any, in the GFL (Fig. 4Ci, Cii). Signals from both of these probes are far above the background level shown by control sections hybridized with a sense probe (Figs. $4 D i, D i i)$. Probe for SqKv1D consistently labeled small groups of moderately sized SG neurons, but labeling intensity was always weak (Fig. 4E). This field is from an area corresponding to that in Figure 4Dii taken from a serial section of the same ganglion. Clusters of such neurons were present in various portions of the $\mathrm{SG}$, but none was present in the GFL. SqKv1C mRNA was not detectable with in situ hybridizations.

Regardless of the probe used, hybridization was restricted to cell bodies and never occurred in the SG neuropil, in tracts of fusing axons within the GFL, in the cytoplasm of the giant axons, or in Schwann cell sheaths surrounding axons in the stellar nerves. Thus, mRNA for these Kv1 $\alpha$-subunits seems to be restricted to neuronal cell bodies.

\section{Functional expression in oocytes and comparison to native $K$ currents in squid neurons}

Patch-clamp measurements of voltage-gated K current $\left(I_{\mathrm{K}}\right)$ in oocytes expressing SqKv1A channels and of native delayed rectifier $I_{\mathrm{K}}$ in GFL neurons and giant axons were described by Rosenthal et al. (1996), who proposed that SqKv1A corresponds to the native $20 \mathrm{pS}$ $\mathrm{K}$ channel. In the present study cell-attached patch recordings were performed on oocytes injected with SqKv1A or SqKv1B cRNA to perform a preliminary comparison of properties of $I_{\mathrm{K}}$ for the two cloned channels and to compare SqKv1B currents with $I_{\mathrm{K}}$ recorded from large $\mathrm{SG}$ neurons that seem to express this mRNA. We have not yet expressed SqKv1C or D.

\section{Activation properties}

Macroscopic $I_{\mathrm{K}}$ records from an oocyte expressing SqKv1A channels are shown in Figure $5 A$, and analogous data for SqKv1B are given in Figure $5 B$. SqKv1B currents closely resemble those for
SqKv1A but were expressed routinely at a higher level. Cellattached patch recordings from the somata of a GFL neuron (Fig. $5 C$ ) and large SG neuron (Fig. 5D) are illustrated also. $I_{\mathrm{K}}$ in these cell types, which selectively express these mRNAs, shows obvious similarity to each other and to the respective traces from oocytes.

$\mathrm{K}$ conductance $\left(g_{\mathrm{K}}\right)$ was determined as $\Delta I / \Delta V$ on termination of the activating pulse at the time of peak $I_{\mathrm{K}}$ (see inset to Fig. $5 E$ ), and $g_{\mathrm{K}}-V$ relations for oocytes expressing SqKv1A or SqKv1B are very similar (Fig. $5 E$ ). Each cloned channel displays a $g_{\mathrm{K}}-V$ relation that is displaced by -10 to $-15 \mathrm{mV}$ (at the foot) with respect to data from GFL and SG neurons. This may reflect the different $\mathrm{Ca}$ concentrations in the two sets of experiments $(0 \mathrm{mM}$ in oocytes vs $10 \mathrm{~mm}$ in neurons). Although $g_{\mathrm{K}}-V$ curves from neurons and oocytes begin to rise with similar slopes, native $g_{\mathrm{K}}$ in both types of neurons approaches saturation more gradually than does $g_{\mathrm{K}}$ in oocytes. This result is not attributable to the difference in ionic strength in the two sets of experiments, as demonstrated by the whole-cell data obtained from GFL neurons with solutions of an ionic composition similar, except for the Ca level, to those for oocyte recordings (asterisk in Fig. $5 E$ ).

Activation kinetics were assessed as the time to reach $50 \%$ of peak $I_{\mathrm{K}}\left(t_{1 / 2}\right)$, and the voltage dependence of $t_{1 / 2}$ is established in Figure $5 F$ for oocytes expressing $\mathrm{SqKv} 1 \mathrm{~A}$ and $\mathrm{B}$ as well as for GFL and large SG neurons. $I_{\mathrm{K}}$ in oocytes activates somewhat more slowly at negative voltages than that in neurons, but the difference is not large.

\section{Deactivation properties}

Although activation properties of SqKv1A and B channels are fairly comparable to those of the native channels in GFL and SG neurons, the rates of channel closing (deactivation) are markedly different. Figure $6 A$ shows tail currents for SqKv1B recorded at a series of voltages after a brief pulse to $+40 \mathrm{mV}$. Closing kinetics are voltage-dependent but much slower than those observed in either large SG neurons (Fig. 6B) or GFL neurons (Fig. 6C). Figure $6 D$ illustrates the voltage dependence of deactivation kinetics $\left(\tau_{\text {OFF }}\right)$, obtained by fitting a single exponential to tail currents. A striking difference between the cloned and native channel types is evident.

\section{DISCUSSION}

This paper describes four closely related SqKv1 mRNAs, three of which show distinct spatial patterns of expression in the SG/GFL complex. SqKv1A is expressed exclusively in the monotypic pool of GFL neurons that forms the giant axon system and represents the only Kv1 mRNA we have detected in these neurons. SqKv1B is expressed abundantly in many neurons of the SG proper, but not in the GFL. Larger SG neurons expressing SqKv1B mRNA undoubtedly include the cell bodies of the small axon motor system. SqKv1D is expressed in isolated small clusters of SG neurons but apparently not at all in GFL neurons.

Our attention thus far has focused on the SG/GFL complex, a peripheral motor control center. We do not know whether SqKv1 mRNAs are expressed elsewhere in the nervous system. Preliminary evidence from RNase protection assays and in situ hybridizations suggests that $\mathrm{SqKv1B}$ is expressed in the optic lobe, but neither SqKv1A nor D mRNA was detectable. We have not yet explored other regions of the CNS.

\section{Alternative splicing of SqKv1A-D mRNAs?}

Although individual SqKv1 mRNAs are expressed at different levels in distinct cell types, their structures are extremely similar. 

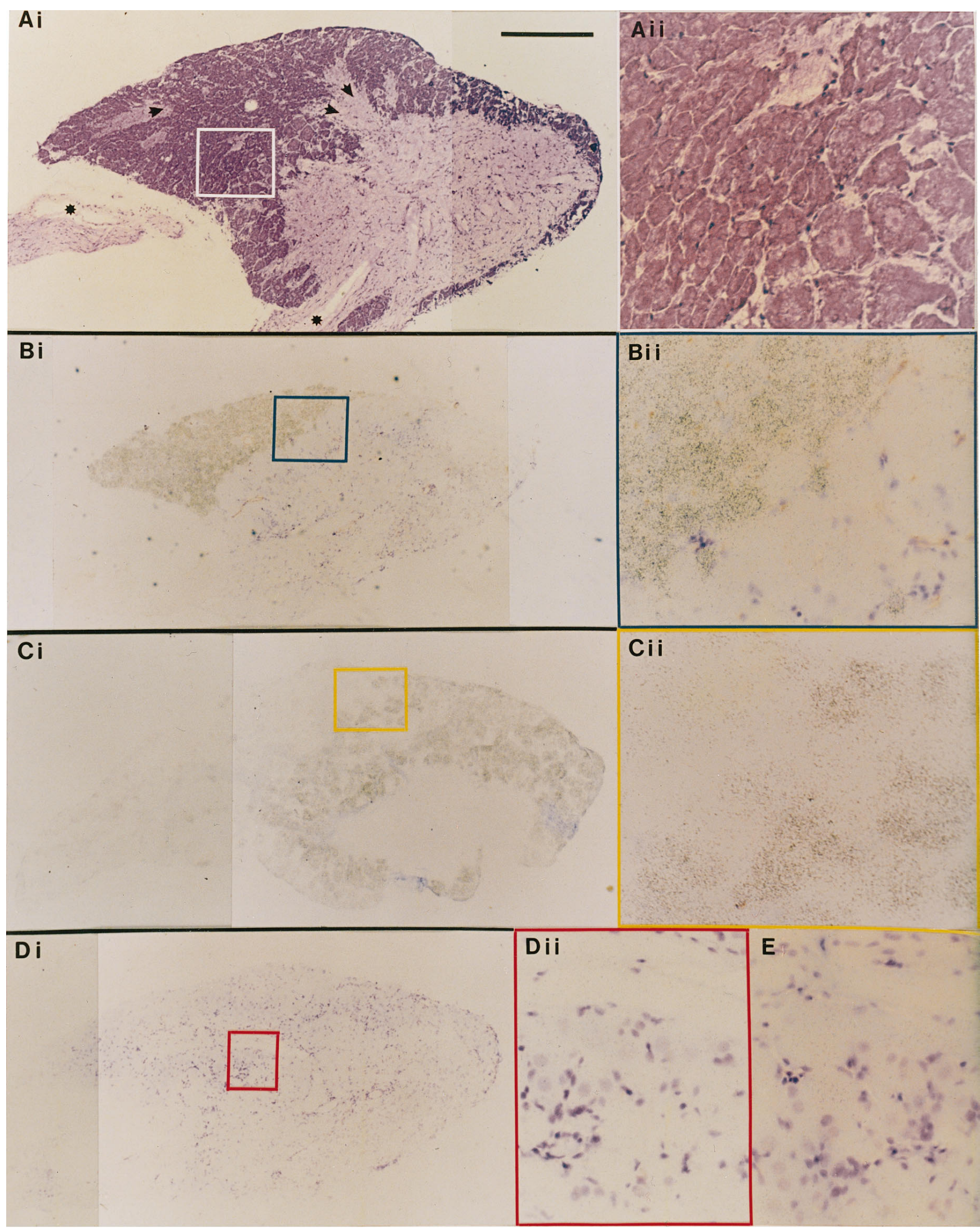

\section{Bi i}


A
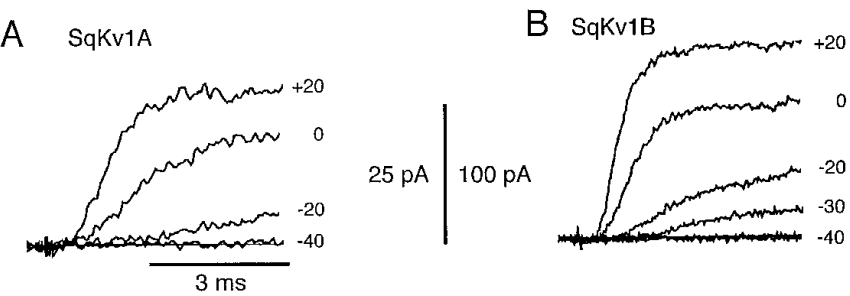

C
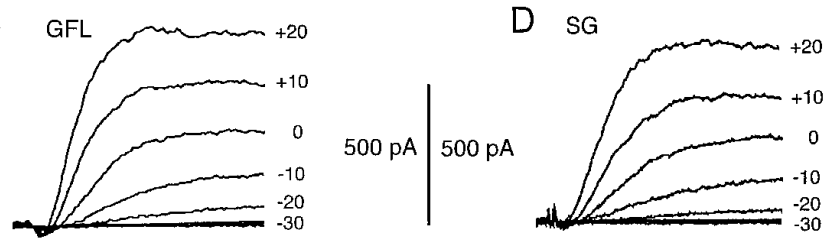

E

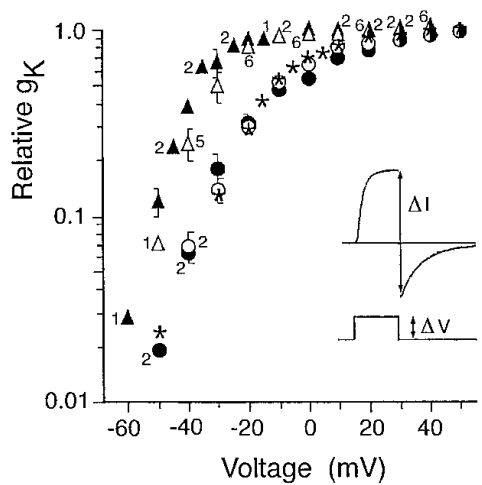

$\mathrm{F}$

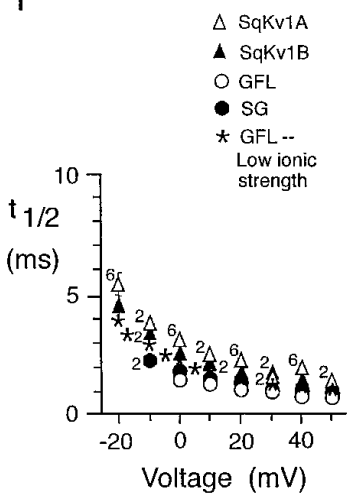

Figure 5. Activation properties of macroscopic $I_{\mathrm{K}}$ recorded from oocytes and squid neurons. Cell-attached patch currents are illustrated at the indicated voltages from oocytes injected with cRNA for $\operatorname{SqKv} 1 \mathrm{~A}(A)$ or SqKv1B $(B)$ and from cell bodies of GFL neurons $(C)$ and large SG neurons $(D)$. The general form of $I_{\mathrm{K}}$ is similar in all cases. $E$, Comparison of voltage dependence of $g_{\mathrm{K}}$ for cloned and native K channels. Relative $g_{\mathrm{K}}$ was estimated as $\Delta I / \Delta V$ after repolarization at the time of peak $I_{\mathrm{K}}$ (see inset). Symbol code is indicated on the figure. Plotted points are mean values $\pm 1 \mathrm{SEM}$. The number of patches $(n)$ is indicated, except where $n=$ 3 or 4. Error bars are omitted when smaller than the symbol or when $n<$ 3. F, Activation kinetics $\left(t \frac{1}{2}\right)$ were assayed as the time to reach $50 \%$ peak $I_{\mathrm{K}}$. Symbols are plotted as in $A$.

The $3^{\prime}$ UTR and most coding sequences of the four mRNAs are virtually identical. Major differences only exist upstream of a common point and give rise to distinct $5^{\prime}$ UTR and N-terminal coding regions. This pattern is very suggestive of alternative splicing. We have not isolated genomic clones for these genes, however, so we cannot prove this conjecture. Attempts to amplify genomic sequence spanning the putative splice site (near nt 35 of SqKv1A) with PCR were unsuccessful, and this would be consistent with the presence of a large intron and the possibility of splicing. The nucleotide sequence of SqKv1A, C, and D lying 5' to this site is AG, consistent with the consensus for $5^{\prime}$ exons. SqKv1B nucleotide sequence is $\mathrm{AT}$ at the equivalent positions, but the
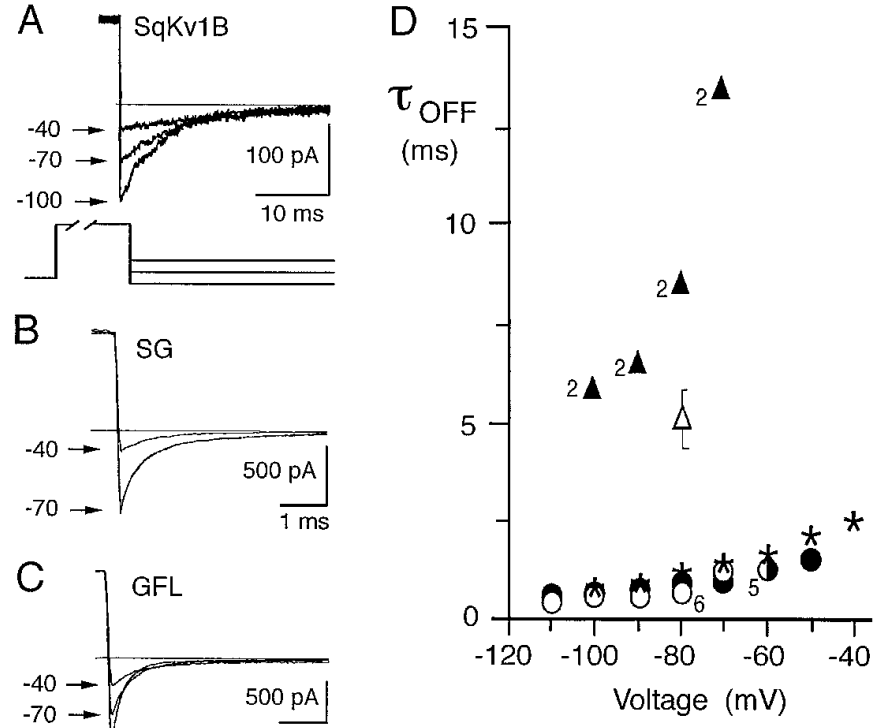

Figure 6. Deactivation properties of macroscopic $I_{\mathrm{K}}$ recorded from oocytes and squid neurons. $A$, Tail currents in a patch from an oocyte injected with SqKv1B cRNA were measured at the indicated voltages after a $25 \mathrm{msec}$ test pulse (see inset). $B$, Analogous tail current records from a patch on a large SG neuron. Note the difference in time scale from $A$. $C$, Tail currents from a patch on a GFL neuron. $D$, Deactivation kinetics $\left(\tau_{\mathrm{OFF}}\right)$ were obtained by fitting a single exponential to the tail current after repolarization of a brief test pulse (usually $25 \mathrm{msec}$ duration). Symbols are plotted as in Figure 5.

consensus sequence for $5^{\prime}$ exons is not conserved absolutely (Lewin, 1994).

Extensive alternative splicing of the single Shaker Kv1 gene occurs in Drosophila (Papazian et al., 1987; Kamb et al., 1988; Pongs et al., 1988; Schwarz et al., 1988), but splicing of mRNAs derived from the multiple Kv1 genes characteristic of other phyla seems to be far more limited (Attali et al., 1993; Jegla et al., 1995). Shaker A-D show $5^{\prime}$ and $3^{\prime}$ splicing, which results in $\alpha$-subunits with distinct $\mathrm{N}$ and $\mathrm{C}$ termini. Different splice variants are expressed in specific neural tissues (Schwarz et al., 1990; TsengCrank et al., 1991), and tissue-specific 3' splicing has been demonstrated in the nervous system (Mottes and Iverson, 1995). Physiological properties of Shaker channels encoded by these splice variants differ in regard to inactivation, with $5^{\prime}$ variants differing in N-type inactivation and $3^{\prime}$ variants differing in C-type (Timpe et al., 1988; Hoshi et al., 1990, 1991; Iverson and Rudy, 1990; Mottes and Iverson, 1995).

Differences in the $\mathrm{N}$ termini of SqKv1A and B channels, on the other hand, do not seem to affect inactivation properties in oocytes (Rosenthal et al., 1996; our unpublished data), nor do any of the SqKv1 $\alpha$-subunits display an "inactivation ball" motif like that of Shaker variants (Murrell-Lagnado and Aldrich, 1993). Furthermore, $I_{\mathrm{K}}$ in GFL neurons and large SG neurons, the cell types expressing the relevant mRNAs, shows no obvious differences in

specific for SqKv1A detects signal only from GFL neurons. Bii, Higher magnification of the boxed region in Bi enclosing the SG/GFL boundary. Virtually no cells are labeled in the SG proper, although a very small number of cells near the boundary seem to express SqKv1A. One such cell is visible in the bottom right corner. Ci, In situ hybridization of ${ }^{35} \mathrm{~S}$-labeled cRNA probe specific for SqKv1B detects signal almost exclusively in SG neurons. Cii, Higher magnification of the boxed region in $\mathrm{Ci}$ enclosing the SG/GFL boundary. Several very large SG neurons are heavily labeled. Di, Control in situ hybridization of ${ }^{35}$ S-labeled cRNA sense probe. Dii, Higher magnification of the boxed region in Di. This region of the SG proper includes neuropil (bottom right) and tracts of small axons projecting from the GFL into the SG (top). E, In situ hybridization of ${ }^{35}$ S-labeled cRNA probe specific for SqKv1D of a SG region equivalent to that in Dii. Tracts of GFL axons are at the top. A cluster of fairly large neurons in the center of the picture is labeled above background and lies just below these axons. 
inactivation properties. These observations are consistent with the idea that neither SqKv1A nor B possesses N-type inactivation analogous to that in Shaker, but the mechanism of inactivation in the cloned squid channels has not yet been studied thoroughly. Inactivation of delayed rectifier $I_{\mathrm{K}}$ in GFL neurons and giant axons is unusually complex but shows more similarity to a conventional C-type process than to an N-type one (Mathes et al., 1997).

\section{Isolated point differences in SqKv1A-D mRNAs}

If differences in 5' UTR and N-terminal coding sequences among SqKv1A-D are attributable to alternative splicing, what might be the basis of the scattered single nucleotide differences? It is very unlikely that the point differences are attributable to random sequencing errors, because they occur at discrete sites and 6 of the 10 have been verified in 19 independent SqKv1A and 9 SqKv1B clones (T3-SKC9 amplification products). One possibility is that these reflect allelic variations among individual squid, because our cDNA library was prepared from pooled SG of many individuals. Although these squid all were captured from a single school in the wild, individual or even population differences could exist. The fact that every difference was an A-G exchange is not consistent with normal patterns of allelic variation, however. An alternative is that these differences reflect adenosine deamination because of mRNA editing (Scott, 1995). A similar pattern in squid Kv2 mRNAs has been attributed to this mechanism (Patton and Bezanilla, 1996), but editing of SqKv1 mRNAs must, at this time, be regarded as speculative.

\section{Functional differences between SqKv1 channels in oocytes and native channels in neurons}

Macroscopic $I_{\mathrm{K}}$ properties derived from oocytes expressing SqKv1A and B are generally comparable to those from GFL and large SG neurons. Limited single channel for SqKv1B in oocytes (our unpublished data) also revealed properties comparable to those reported for SqKv1A (Rosenthal et al., 1996). Thus, unitary conductance for both channels is $\sim 12 \mathrm{pS}$ measured in oocyte solutions $(\sim 20 \mathrm{pS}$ when adjusted for the higher $\mathrm{K}$ concentration in squid solutions), and both channels open with short latency, show prominent bursting, and inactivate over several hundred milliseconds. These properties are shared by the $20 \mathrm{pS}$ channels in giant axons and GFL somata.

Some significant differences in macroscopic properties exist, however, between the cloned and native delayed rectifier channels. The major differences are the steeper overall rise of the $g_{\mathrm{K}}-V$ curve and the unusually slow deactivation kinetics shown by SqKv1A and B. These differences do not reflect a gross difference in ionic strength or composition of oocyte versus squid salines, but a precise comparison will require comparisons under identical ionic conditions, probably with excised patch recordings. It is also unlikely that the large differences reflect contamination of native $I_{\mathrm{K}}$ by additional channel types, particularly in GFL neurons that have been well studied in this regard (see introductory remarks).

Although we have attempted to account for the Kv1 mRNAs expressed in the SG/GFL complex and find that SqKv1A and B are by far the predominant species, we cannot rule out the possibility that either of these $\alpha$-subunits might form heteromultimeric channels in squid neurons, thereby conferring unique functional properties on the native channels. For example, SqKv1D $\alpha$-subunits (which we have not yet been able to express) might assemble with SqKv1B in a very small minority of SG neurons, but the chances of routinely encountering such cells in our recordings would seem very remote. Similarly, the existence of undiscovered $\alpha$-subunits that can assemble with either SqKv1A or $\mathrm{B}$ is difficult to disprove.

If SqKv1A and B mRNAs alone encode the native channels in GFL and large SG neurons, the idea we favor, then particular functional properties must be influenced by factors other than primary structure of the $\alpha$-subunit. Many potential mechanisms could be relevant, including post-translational processing of some or all of the $\alpha$-subunits, interactions with other cellular components such as $\mathrm{Kv} \beta$-subunits, or even differences in membrane composition. Such factors are likely to differ substantially in squid neurons and frog oocytes, and accessibility of the native cell types in squid may expedite identification of modulatory influences that are important to in vivo operation of molecularly identified channels.

\section{Biological importance of SqKv1A-D mRNAs and channels}

Results described in this work are consistent with the idea that the molecular complexity of the Kv1 $\alpha$-subunit complement in the giant axon system is rather simple (Rosenthal et al., 1996) and suggest that a similar situation may exist for many of the larger SG neurons that express SqKv1B. If both of these cell types support long motor axons and rapid transmission of brief impulses in high frequency bursts (Otis and Gilly, 1990), why should distinct mRNAs exist to express $\mathrm{K}$ channels with such similar functional properties?

This question also applies to other taxa, including vertebrates, and the answer presently is unknown. The unique $\mathrm{N}$ terminals of SqKv1A and B $\alpha$-subunits, or the anomalous residues in the NAB domain, could lead to important differences in interactions with other cellular proteins that also may be specific to individual types of neurons. Such interactions could affect channel localization or functional properties. Although properties of $\mathrm{K}$ channels in the somata of GFL cells and large SG neurons are indistinguishable, differences in their dendrites, motor terminals, or even axons might exist.

Finally, GFL neurons represent a highly specialized pool of monotypic cells that may express many unique genes. Studies of the distinct 5' UTRs of the SqKv1 mRNAs expressed in the SG/GFL complex might lead to a deeper understanding of the importance of these elements in regulating expression of specific channel subtypes in certain neurons. Our understanding of differentiation in GFL neurons and development of the giant axon is very limited (Gilly et al., 1991), and SqKv1A-specific probes will provide an important tool to examine these processes.

\section{REFERENCES}

Attali B, Lesage F, Ziliani P, Guillemare E, Honore E, Waldmann R, Hugnot JP, Mattei MG, Lazdunski M, Barhanin J (1993) Multiple mRNA isoforms encoding the mouse cardiac $\mathrm{Kv} 1-5$ delayed rectifier $\mathrm{K}^{+}$channel. J Biol Chem 268:24283-24289.

Dalbey RE, Von Heinje G (1992) Signal peptidases in prokaryotes and eukaryotes: a new protease family. Trends Biochem Sci 17:474-478.

Drewe JA, Verma S, Frech G, Joho RH (1992) Distinct spatial and temporal expression patterns of $\mathrm{K}^{+}$channel mRNAs from different subfamilies. J Neurosci 12:538-548.

Gilly WF, Lucero MT, Horrigan FH (1990) Control of the spatial distribution of sodium channels in giant fiber lobe neurons of the squid. Neuron 5:663-674.

Gilly WF, Hopkins B, Mackie GO (1991) Development of giant motor axons and neural control of escape responses in squid embryos and hatchlings. Biol Bull 180:209-220.

Gilly WF, Preuss T, McFarlane MB (1996) All-or-none contraction and sodium channels in a subset of circular muscle fibers of squid mantle. Biol Bull 191:337-340. 
Hoshi TW, Zagotta WN, Aldrich RW (1990) Biophysical and molecular mechanisms of Shaker potassium channel inactivation. Science 250:533-538.

Hoshi TW, Zagotta WN, Aldrich RW (1991) Two types of inactivation in Shaker K channels: effects of alterations in the carboxy-terminal region. Neuron 7:547-556.

Iverson LE, Rudy B (1990) The role of divergent amino and carboxy domains on the inactivation properties of potassium channels derived from the Shaker gene of Drosophila. J Neurosci 10:2903-2916.

Jan LY, Jan YN (1992) Structural elements involved in specific $\mathrm{K}^{+}$ channel functions. Annu Rev Physiol 54:537-555.

Jegla T, Grigoriev N, Gallin W, Salkoff L, Spencer AN (1995) Multiple Shaker potassium channels in a primitive metazoan. J Neurosci 15:7989-7899.

Kamb A, Tseng-Crank J, Tanouye MA (1988) Multiple products of the Drosophila Shaker gene may contribute to potassium channel diversity. Neuron 1:421-430.

Kozak M (1989) The scanning model for translation: an update. J Cell Biol 108:229-244.

Lewin B (1994) Genes, Vol V (Lewin B, ed), p 914. Toronto: Oxford UP.

Li M, Jan YN, Jan LY (1992) Specification of subunit assembly by the hydrophilic amino-terminal domain of the Shaker potassium channel. Science 257:1225-1230.

Liu TI, Gilly WF (1995) Tissue distribution and subcellular localization of $\mathrm{Na}^{+}$channel mRNA in the nervous system of the squid, Loligo opalescens. Receptors Channels 3:243-245.

Llano I, Bookman RJ (1986) Ionic conductances of squid giant fiber lobe neurons. J Gen Physiol 88:543-569.

Llano I, Webb CK, Bezanilla F (1988) Potassium conductance of the squid giant axon: single channel studies. J Gen Physiol 92:179-196.

Mathes C, Rosenthal JJC, Armstrong CM, Gilly WF (1997) Fast inactivation of the delayed rectifier potassium conductance in squid giant axon and its cell bodies. J Gen Physiol 109:435-448.

Murrell-Lagnado RD, Aldrich RW (1993) Interactions of amino terminus domains of Shaker $\mathrm{K}$ channels with a pore-blocking site studied with synthetic peptides. J Gen Physiol 103:949-975.

Mottes JR, Iverson LE (1995) Tissue-specific alternative splicing of hybrid Shaker/lacZ genes correlates with kinetic differences in Shaker $\mathrm{K}^{+}$ currents in vivo. Neuron 14:613-623.

Nealey TS, Spires S, Eatock RA, Begenisich T (1993) Potassium channels in squid neuron cell bodies: comparison to axonal channels. J Membr Biol 132:13-25.

Otis TS, Gilly WF (1990) Jet-propelled escape in the squid Loligo opalescens: concerted control by giant and non-giant motor axon pathways. Proc Natl Acad Sci USA 87:2911-2915.

Papazian DM, Schwarz TL, Tempel BL, Jan YN, Jan LY (1987) Cloning of genomic and complementary DNA from Shaker, a putative potassium channel gene from Drosophila. Science 237:749-753.

Patton DE, Bezanilla F (1996) RNA editing generates diverse transcripts encoding squid Kv2 $\mathrm{K}^{+}$channels. Biophys J 70:A13.

Perozo E, Jong DS, Bezanilla F (1991) Single channel studies of the phosphorylation of $\mathrm{K}^{+}$channels in the squid giant axon. II. Nonstationary conditions. J Gen Physiol 98:19-34.

Pongs O (1992) Molecular biology of voltage-dependent potassium channels. Physiol Rev 72:S69-S88.
Pongs O, Kecskemethy N, Muller R, Krah-Jentgens I, Baumann A, Kiltz HH, Canal I, Llamazares S, Ferrus A (1988) Shaker encodes a family of putative potassium channel proteins in the nervous system of Drosophila. EMBO J 7:1087-1096.

Prosser CL, Young JZ (1937) Responses of muscles of the squid to repetitive stimulation of the giant nerve fibres. Biol Bull 73:237-241.

Rosenthal JJC, Gilly WF (1993) Amino acid sequence of a putative sodium channel expressed in the giant axon of the squid Loligo opalescens. Proc Natl Acad Sci USA 90:10026-10030.

Rosenthal JJC, Vickery RG, Gilly WF (1996) Molecular identification of SqKv1A: a candidate for the delayed rectifier $\mathrm{K}$ channel in squid giant axon. J Gen Physiol 108:207-219.

Sambrook J, Fritsch EF, Maniatis T (1989) Molecular cloning: a laboratory manual. Cold Spring Harbor, NY: Cold Spring Harbor Laboratory.

Schwarz TL, Tempel BL, Papazian DM, Jan YN, Jan LY (1988) Multiple potassium channel components are produced by alternative splicing at the Shaker locus in Drosophila. Nature 331:137-142.

Schwarz TL, Papazian DM, Carretto RC, Jan YN, Jan LY (1990) Immunological characterization of $\mathrm{K}^{+}$channel components from the Shaker locus and differential distribution of splicing variants in Drosophila. Neuron 2:119-127.

Scott J (1995) A place in the world for RNA editing. Cell 81:833-836.

Shen NV, Pfaffinger PJ (1995) Molecular recognition and assembly sequences involved in the subfamily-specific assembly of voltage-gated $\mathrm{K}^{+}$ channel subunit proteins. Neuron 14:625-633.

Sewing S, Roeper J, Pongs O (1996) Kv $\beta 1$ subunit binding specific for Shaker-related potassium channel $\alpha$-subunits. Neuron 16:455-463.

Simmons DM, Arriza JL, Swanson LW (1989) A complete protocol for in situ hybridization of messenger RNAs in brain and other tissues with radiolabeled single-stranded RNA probes. J Histotechnol 12:168-181.

Stuhmer W, Ruppersberg JP, Schroter KH, Sakmann B, Stocker M, Giese KP, Perschke A, Baumann A, Pongs O (1989) Molecular basis of functional diversity of voltage-gated potassium channels in mammalian brain. EMBO J 8:3235-3244.

Timpe LC, Jan YN, Jan LY (1988) Four cDNA clones from the Shaker locus of Drosophila induce kinetically distinct A-type potassium currents in Xenopus oocytes. Neuron 1:659-667.

Tseng-Crank J, Pollock JA, Hayashi I, Tanouye MA (1991) Expression of ion channel genes in Drosophila. J Neurogenet 7:229-239.

Von Heinje G (1990) Protein targeting signals. Curr Opin Cell Biol 2:604-608.

Wilson DM (1960) Nervous control of movement in cephalopods. J Exp Biol 37:57-72.

Xu J, Yu W, Jan YN, Jan LY, Li M (1995) Assembly of voltage-gated potassium channels: conserved hydrophilic motifs determine subfamily-specific interactions between the $\alpha$-subunits. J Biol Chem 270:24761-24768.

Young JZ (1938) The functioning of giant nerve fibres of the squid. J Exp Biol 15:171-185.

Young JZ (1939) Fused neurons and synaptic contacts in the giant nerve fibres of cephalopods. Phil Trans R Soc Lond [Biol] 229:465-503.

Young JZ (1972) The organization of a cephalopod ganglion. Phil Trans R Soc Lond [Biol] 263:409-429.

Yu W, Xu J, Li M (1996) NAB domain is essential for the subunit assembly of both $\alpha-\alpha$ and $\alpha-\beta$ complexes of Shaker-like potassium channels. Neuron 16:441-453. 